\title{
PROJECT FOR GENERATION OF FEMTOSECOND X-RAY BEAMS FROM THE DUKE STORAGE RING *
}

\author{
V.N.Litvinenko, O.A.Shevchenko, S.F.Mikhailov, FEL Laboratory, Department of Physics, Duke \\ University, Durham, NC 27708, U.S.A \\ Y. Wu, Lawrence Berkeley National Laboratory, 1 Cyclotron Road, Berkeley, CA 94720, USA
}

\section{Abstract}

We propose to develop a CW X-ray femtosecond (fsec) source at $1.2 \mathrm{GeV}$ Duke storage ring with the X-ray energy tuneable from $1.4 \mathrm{KeV}$ to $75 \mathrm{keV}$, the average flux from $10^{12}$ to $10^{13} \mathrm{ph} / \mathrm{sec}$, and the average spectral brightness up to $0.510^{15} \mathrm{ph} / \mathrm{sec} / \mathrm{mm}^{2} / \mathrm{mrad}^{2} / 0.1 \% \mathrm{BW}$. A novel RF system with strong longitudinal focusing will compress and to sustain the electron bunches circulating in the ring to a RMS duration from 15 to $85 \mathrm{fsec}$. The $\mathrm{CW}$ fsec electron bunches will generate the Compton hard-Xray beams and the photon beams from the existing light sources ranging form IR to VUV. Beams of all colours will be synchronised by the e-beam without jitter, making this configuration perfectly suites for pump-probe experiments.

The proposed approach promises to outperform the popular "slicing technique" by at least three orders of magnitude. Duke's approach can be implemented at existing light source facilities at modest cost.

\section{INTRODUCTION}

Development of hard X-ray beams with fsec duration is the unrivaled frontier for modern light sources. Several national laboratories developing large-scale projects based on X-ray FELs (LCLS at Stanford, TESLA X-ray FEL at DESY [3]) or prototype systems with low intensity beams (for example, beam slicing at LBNL, [2]). At Duke, we have identified an alternative approach, unique and remarkably effective, which can be used at any synchrotron radiation source for generation of hard X-ray beams with fsec duration. The key is the strong longitudinal focusing using an advanced mm-wave RF system, an inverse mm-wave FEL, to compress and to stabilize short electron bunches $[1,4]$. The power for mmwave RF system will be provided by a stand-alone mmwave FEL based on the re-circulating accelerator, a scaled down version of the known and tested technology [5].

The circulating fsec electron bunches in the storage ring will generate the full spectrum of intense light, from Xrays to the IR and will imprint their fsec time structure into all short-wavelength light beams without jitter. The hard X-rays with tunable energy will be generated via Compton back-scattering from the same e-beam. The validity and effectiveness of this approach has been demonstrated at DFELL with the production of intense beams of $\gamma$-rays [6]. These conditions are ideal for multicolor pump-probe experiments.

The description and the present performance of the Duke storage ring FEL facility are published elsewhere [7]. In this paper we describe the proposed intense X-ray fsec source. The proposed system will be located in the technical straight section of the Duke ring.

\section{PRINCIPLES OF OPERATION}

The key for the proposed fsec X-ray light source is to create and to sustain electron bunches with fsec duration in the storage ring. In a conventional storage ring [7] the bunch lengthening and the growth of the energy spread caused by the longitudinal wake-fields and by coherent synchrotron radiation (CSR) did not allow to operate with fsec e-bunches (see, for example, [10]). The main obstacle for very short e-bunches is the wake field (to be exact, the forward field) caused by CSR, which exceeds those caused by vacuum chamber by many orders of magnitude (see Fig.1 below).

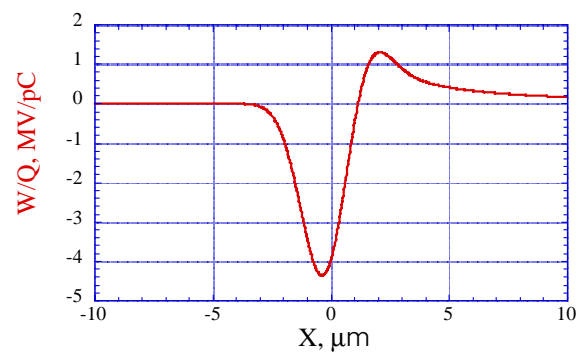

Fig. 1 CSR wakefield for $1 \mathrm{GeV}, 1 \mathrm{pC}$ e-bunch with RMS length of $1 \mu \mathrm{m}$ (3 fsec) in the Duke storage ring.

We found that strong longitudinal focusing based on sub$\mathrm{mm}$ inverse FEL is sufficient for suppressing the microwave instability. Detailed description of the inverse FEL RF system and the concept of strong longitudinal focusing can be found in [1]. The voltage of this novel RF system and the peak FEL power are connected by the following expression [1]:

$$
\hat{P}[M W] \cong 300 \frac{V^{2}[M V]}{N_{w}} e^{\frac{1}{\pi N_{w}}} ;
$$

where $\mathrm{N}_{\mathrm{w}}$ is the number periods in the "RF-wiggler". For the Duke storage ring with $E_{o}=1 \mathrm{GeV}, \mathrm{C}=107.46 \mathrm{~m}, \alpha_{c}$ $=310^{-3}$, it is sufficient to provide a voltage of 
$V_{r f}[M V] \cong \lambda_{r f}[\mathrm{~mm}]$ to get $Q s=0.31$ with the mm-wave RF systems. Detailed studies of fsec e-bunches dynamics give following theoretical stability threshold [9]:

$$
I_{\text {peak }} \sim \frac{16 \pi^{3} E}{e Z\left(c / \sigma_{l}\right)} \cdot \frac{Q_{s}}{\alpha_{c}} \cdot \frac{\sigma_{l}}{C},
$$

where $Z(\omega)=Z_{o} \frac{\Gamma(2 / 3)}{3^{1 / 3}}\left[\frac{\sqrt{3}}{2}+\frac{i}{2}\right]\left(\frac{\omega}{\omega_{o}}\right)^{1 / 3}$ is the CSR impedance [8]. Equation (4) depends on the synchrotron tune and differs from the Boussard's criterion [10]. Self-consistent numerical simulations [10] showed that the criterion (4) underestimates the threshold peak current, higher e-beam currents can be operated with the fsec duration.

\section{NUMERICAL SIMULATIONS}

In order to investigate longitudinal dynamics of fsec electron bunches we developed a self-consistent numerical model and two computer codes for simulations. Electrons in the bunch are represented by macroparticles with equal charges. A macroparticle is described by its energy, longitudinal position, transverse positions and momenta. Each macroparticle is tracked for a large number of turns, while interacting with RF cavities, magnetic lattice, wakefields, and CSR, and is subjected to quantum fluctuations of the synchrotron radiation and radiation damping. The total wakefield is calculated in a number of locations around the ring (as the direct sum of the fields induced by individual macroparticles), and the energy kick is applied to each macroparticle depending on its location in the bunch. To reduce shot noise, we assumed that each macroparticle is a Gaussian cluster with $1 \mu \mathrm{m}$ RMS length.
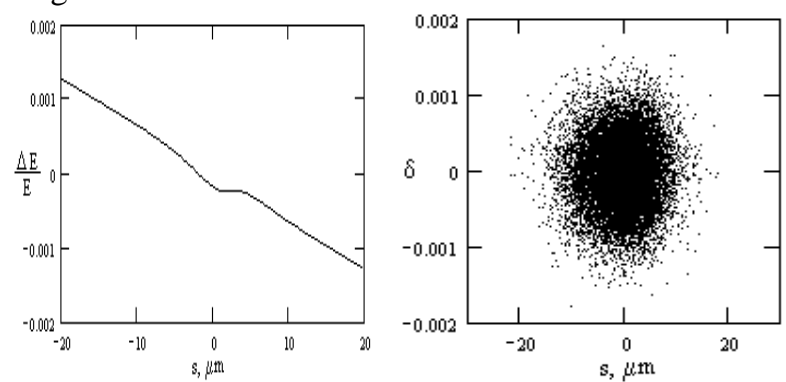

Fig. 2 Stable $750 \mathrm{MeV}, 0.1 \mathrm{pC}$ fsec e-beam. $\mathrm{V}_{0.25 \mathrm{~mm}}=2 \mathrm{MV}$, $\alpha_{c}=0.2510^{-4}, \sigma_{t}=11.2$ fsec, $\sigma_{\mathrm{E}}=0.044 \%$. One turn total energy kick is on the left. Pointcare plot is on the right

We found that simulations of an electron bunch with $0.5 \mathrm{pC}$ charge and RMS bunch length of 15 microns (50 fsec) do not exhibit any energy spread growth or substantial bunch lengthening. Some parameters used in the simulation are listed in Table 1. Fig.2. shows a typical Pointcare plot of a stable e-beam with fsec duration and the resulting energy kick per turn. We found that for the Duke ring the sustainable bunch length can be approximated by the following expression:

$$
\sigma_{\tau}[f \mathrm{sec}] \approx 40\left(\frac{\lambda_{r f}[\mathrm{~mm}]}{V_{r f}[M V]}\right)^{2 / 3} .
$$

Table 1. Parameters used for tracking

Focusing RF ( $\lambda=0.25-1 \mathrm{~mm}), \mathrm{GHz}$

$300-1,200$

Voltage, focusing RF, MV

Orbit compaction factor, $\alpha_{c}$

Number of macro particles, $\mathrm{N}$

Number of turns

Interactions with wake per turn

$0-2$

$8.610^{-3}-110^{-5}$

$1,000-20,000$

$\sim 20,000$

$\sim 16$

\section{FSEC E-BEAM PARAMETERS}

We will use a mode of operation with a very low ebeam currents. Table 2 gives the list of main e-beam parameters.

Table 2. Parameters of the fsec e-beams

\begin{tabular}{ll}
\hline Operating energy, $\mathrm{E}_{\mathrm{e}}[\mathrm{GeV}]$ & $0.25-1.2$ \\
Momentum compaction factor, $\alpha_{\mathrm{c}}$ & $810^{-3}-110^{-5}$ \\
Number of bunches & $1-8$ \\
Multi-bunch beam current $[\mathrm{mA}]$ & $0.01-0.25$ \\
Single bunch beam current $[\mathrm{mA}]$ & $0.001-0.03$ \\
Emittance $(0.75 \mathrm{GeV}), \varepsilon_{\mathrm{x}} / \varepsilon_{\mathrm{y}},[\mathrm{nm} \mathrm{rad}]$, & $10 / 0.5$ \\
Bunch length $[\mathrm{fsec}]$ & $15-100$ \\
\hline
\end{tabular}

\section{MM-FEL SYSTEM}

mm-Wave CW FEL

Recirculator

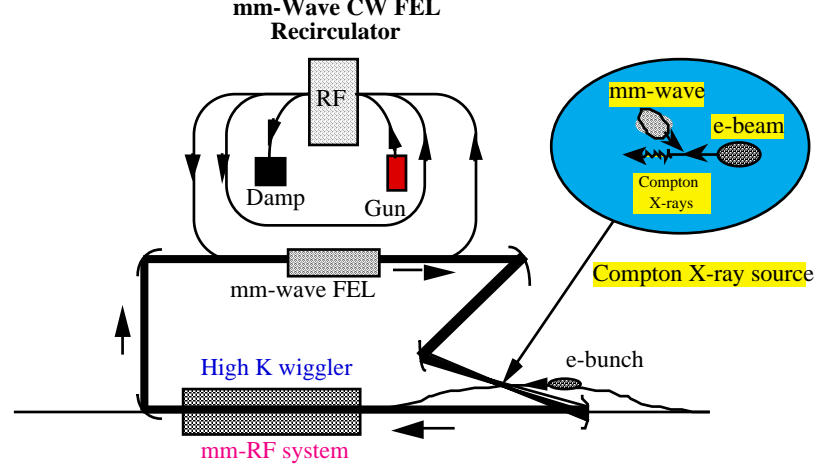

Fig. 3 Sketch of the mm-wave FEL system, the mm-RFsystem and the Compton X-ray source for fsec light source.

The key systems required for the up-grade are sketched in Fig.3. The mm-wave FEL system based on a recirculating 178-MHz RF accelerator. The high intracavity power of this FEL is focused for generation of the hard-Xray beams via Compton back-scattering and later copropagates with the electron beam through a high-K helical wiggler. It acts as a traditional RF system but operates with TEM mm-waves. The circumference optical cavity will be equal to $1 / 8$ of the storage ring circumference to support operating with 8 individual micro-bunches.

A 30-KV DC gun with AC grid will be used as the injector of 1-nsec, $1 \mathrm{~A}$ electron pulses with a rep-rate up to $22.25 \mathrm{MHz}$. After acceleration, these pulses will be compressed to $\sim 0.1 \mathrm{nsec}$ and passed through the mm-wave FEL system. 
Table 3. Parameters of CW mm-wave FEL [11]

\begin{tabular}{ll} 
Energy in FEL, $\mathrm{E}_{\mathrm{e}}[\mathrm{MeV}]$ & $3-6.5$ \\
FEL wavelength, mm & $0.25-1$ \\
Average beam current, A & $0.05-0.1$ \\
Wiggler period, cm & 6,10 \\
Number of periods & 10 \\
FEL gain, \% & $10-20$ \\
FEL efficiency, \% & $2-5$ \\
Energy recovery efficiency, \% & $90-95$ \\
Average FEL power, $\mathrm{kW}$ & $1-10$ \\
Peak intracavity power, GW & $0.1-1$ \\
\hline
\end{tabular}

\section{MM-RF SYSTEM}

The mm-wave FEL will generate the TEM $_{\text {oo }}$ mode, which will be focused into the center of the mm-RF wiggler with a Rayleigh range of $1.5 \mathrm{~m}$ (Table 4). The proposed wiggler will be capable of operating at $\lambda_{\text {rf }}$ of 1.1 $\mathrm{mm}$ and $0.28 \mathrm{~mm}$ with e-beam energies of $0.5 \mathrm{GeV}$ and 1 $\mathrm{GeV}$ respectevly. The use of the helical wiggler increases the efficiency of the interaction and provides a convenient way of avoiding the synchrotron radiation along the beam axis. This mm-RF sistem will provide $0.95 \mathrm{MV}$ voltage at $\mathrm{P}_{\text {peak }}=0.1 \mathrm{GW}$ and , $3 \mathrm{MV}$ at $\mathrm{P}_{\text {peak }}=1 \mathrm{GW}$, correspondently.

Table 4. Parameters of helical electromagnetic wiggler $\begin{array}{lll}\text { Total length }[\mathrm{m}] & 3.0 & \text { Number of periods: }\end{array}$ Period, $\lambda_{\mathrm{w}}[\mathrm{m}] \quad 1.0 \quad$ Aperture $[\mathrm{cm}] \quad 16$ Peak field [kGs] $0-5 \quad \mathrm{~K}_{\underline{w}} \quad 0-46.5$

\section{THE FSEC X-RAY BEAMS}

As soon as the first fsec e-beams are generated, we will use soft X-ray radiation from the bending magnets at 1 GeV (Table 5).

Table 5. Fsec X-ray beam from the bending magnet

Critical wavelength of radiation, $\AA \quad 11.7$

Number of bunches $\quad 1-8$

X-ray flux [photons per sec]

Flux [photons/sec/mrad/0.1\%BW]

$(0.3-6) 10^{13}$

$(0.05-1) 10^{11}$

The Compton Hard-X-ray source (Table 6) is the most straightforward part of the proposed up-grade. The optimization requires a sharply focused mm-wave beam with the Rayleigh range of $1 \mathrm{~cm}$ and the electron beam with $\beta_{\mathrm{x}}=0.1 \mathrm{~m}$ and $\beta_{\mathrm{y}}=0.08 \mathrm{~m}$ at the collision point. The wavelength of the backscattered X-ray photons is well known:

$$
\lambda_{X-r a y}=\frac{\lambda_{m m}}{4 \gamma^{2}}
$$

Table 6. Parameters of fsec hard X-ray source

\begin{tabular}{ll}
\hline X-ray energy [keV] & $1.4-75$ \\
Flux [photons $/ \mathrm{sec}]$ & $0.1-1.210^{12}$ \\
Brightness $\left[\mathrm{ph} / \mathrm{sec} / \mathrm{mm}^{2} / \mathrm{mrad}^{2} / 0.1 \% \mathrm{BW}\right]$ & $0.4-510^{14}$ \\
\hline
\end{tabular}

\section{CONCLUSSIONS}

The proposed up-grades of the Duke storage ring will completed in three years after availability of funds. The Duke storage ring, operating with total e-beam current of $100 \mu \mathrm{A}$, will generate fsec hard X-rays with flux and brightness exceeding that of the "slicing" $\mathrm{X}$-ray source at ALS (LBNL) by a factor of $10^{3}$ and $10^{6}$ times respectively. The best performance for the Duke source will be with 0.5 to $0.8 \mathrm{GeV}$ e-beam and $0.5 \mathrm{~mm}$ waves generating $8 \mathrm{keV}$ to $25 \mathrm{keV} \mathrm{X}$-rays.

Authors are grateful to Bernt Müller and Glenn Edwards, Duke University, for their support and encouragement. Authors would like to thank Brian Kincaid (LBNL) for fruitful discussions of the applicability of fsec X-ray beams.

\section{REFERENCES}

[1] V.N.Litvinenko, AIP 395 (1997) 275; SPIE. 4031 (2000) 254

[2] A.A.Zholentz et al., Phys.Rev.Let. 76, (1996) 912, R.W.Schoenlein at al., Science 287 (2000) 2237

[3] LCLS, The First Experiments, SLAC Report, 2000 B.Faatz et al., NIM A 407 (1998) 302

[4] V.N.Litvinenko, Y.Wu, APAC'98, Tsukuba, March 1998, p. 831,

Y.Wu, V.N.Litvinenko "Longitudinal Dynamics of femtosecond e-beam in the storage ring", Presented at PAC99, (1999) March 1999, New York City, NY; "On possibility of femtosecond e-beam in the storage rings" Presented at PAC'97, and FEL'98.

[5] N.G.Gavrilov at al, IEEE J. QE-27 (1991) 2626 G.R.Neil et al., NIM A 445 (2000) 192

[6] V.N.Litvinenko et al., Physics Review Letters 78 (1997) 4569, NIM. A358 (1995) 345

[7] V.N. Litvinenko et al., "Performance of the OK4/Duke storage ring FEL", accepted for NIM A

[8] K.Bane, wt al., AIP Proc. 367 (1999) 191 J.B.Murphy, S.Krinsky, NIM A 346 (1994) 571

[9] V.N.Litvinenko, O.A.Shevchenko, Y.Wu, "Physics of femtosecond electron beams in storage rings", in preparation for Phys. Rev.

[10] D.Boussard, CERN LABII/rf/INT/75-2 (1975)

[11] V.N.Litvinenko, S.F.Mikhailov, "CW mm-wave FEL - design parameters", Duke FEL Lab, report, Jan. 2001 